\title{
CRIMSONpublishers
}

http://www.crimsonpublishers.com

\section{Biomarker-Guided Nutritional Intervention to Treat Autism}

\author{
Afaf K El-Ansary* \\ Central Laboratory, King Saud University, Kingdom of Saudi Arabia
}

*Corresponding author: Afaf K El-Ansary, Senior Scientist, Central Laboratory, King Saud University, P.0 Box 22452, Zip Code 1149, Riyadh, Kingdom Of Saudi Arabia

Submission: September 13, 2017; Published: November 08, 2017

\section{Letter to Editor}

Autism as a neuro developmental chronic disorder with dramatic increasing prevalence is a great burden on patients and families. Although the Food and Drug Administration (FDA) has never approved a drug as useful in autism, and claims, that nutritional supplements are "unproven" and those parents who use these supplements for their autistic children are the victims of "marketers". I am writing to discuss with you and with the NTNF readers that understanding the etiological mechanisms in autism and recording multiple biomarkers related to these etiologies can be used as target for nutritional intervention strategies.

Of course, it is well accepted that there are no autism-defining, metabolic biomarkers, but investigation of the biomarkers of selected pathways associated with the etiology of autism such as oxidative stress, glutamate excitotoxicity, neuroinflammation, impaired fatty acids and energy metabolism, and excess propionic acid as a metabolite of Clostridium species can point to potentially treatable abnormalities and provide a baseline that can be tracked to help autistics and ameliorates the severity of the disorder. Up to our research team interest in the field of biomarkers, oxidative stress, poor detoxification, immunologic/inflammation, and mitochondrial abnormalities were repeatedly recorded as etiological mechanisms of autism. In addition, there is accumulating evidence on the impairment of lipid metabolism, and abnormal gastrointestinal microbiota. Many studies support the role of the gastrointestinal (GI) microbiota and their fermentation products in the etiology of autism, and suggested that GI-related biomarkers could potentially enable early identification of autism, and thereby help targeted interventions to improve the health and quality of life of autistic patients [1].

Based on our understanding, all these affected pathways are interconnected with a defect in one likely leading to the impairment of others. Mitochondrial dysfunction, impaired detoxification, metabolic imbalances such as impaired GSH/GSSG, Omega-3/omega-6, glutamate/GABA, glutamate/glutamine, and genetic vulnerability together can all lead to oxidative stress as a pathological pathway related to many diseases among which is autism. In turn oxidative stress leads to inflammation, damaged cell membranes, cell death, and neurological dysfunction [2].

Due to the complexity of the brain as the most affected organ in autism, it is accepted that a panel of markers are required rather than one single marker, in order to improve their sensitivity and specificity. Through the use of, multivariate data analysis, combined Receiver Operating Characteristics (ROC) and Principal components analysis PCA) as statistical tools, number of panels related to oxidative stress, neuroinflammation, DNA methylation, glutamate excitotoxicity, impaired fatty acids and energy metabolism were recorded [3-6]. I think that, collaboration between researchers, caregivers, and stakeholders can help to identify valid panel of markers which is clinically useful to guarantee that autistic children and their families are treated with great respect. Validating these panels for use in clinical practice could potentially help in the early diagnosis and nutritional intervention as the most safe and noninvasive treatment strategy.

Complementary alternative medicine (CAM) and nutritional treatments are usually recommended to avoid the side effects of conventional drugs, and to ameliorate the core symptoms of autism. Based on our understanding of the role of gut-brain axis and bacterial and fungal metabolites in the pathology of autism, the administration of safe probiotics (e.g. Bifidobacterium and Lactobacillus) or prebiotics such as fructooligosaccharide (FOS) or galactooligosaccharide (GOS), may provide an excellent tool to treat this disorder. FOS is naturally found in garlic, bananas, and wheat, whereas GOS is enzymatically produced from lactose. Studies have shown the ability of FOS and GOS to induce the growth of Bifidobacterium and Lactobacillus, and to ameliorate glutamate excitotoxicity $[7,8]$.

Here I will give few selected recent examples of nutritional interventions that might help to accept dietary supplements as treatment strategy of autism. In relation to oxidative stress, abnormal serotonin and dopaminergic neurochemistry, and imbalanced Omega-3/omega-6 ratio as biomarkers of autism, omega-3 supplementation can be used as an effective treatment 
strategy. It was effective in treating the aggression and impulsivity in autistic patients. The underlying mechanism of action is not fully understood but may be related to its antioxidant effect, reduction of the level of IL- 6 as a pro-inflammatory cytokine, and the modulation of serotonergic and dopaminergic altered neurotransmission $[9,10]$.

Most recently, both folate and B12 was used to correct mitochondrial dysfunction through the modulation of complex I, complex IV, and citrate synthase as important components of the respiratory chain and citric acid cycle respectively [11]. The rationale for the use of $\mathrm{N}$-acetyl cysteine (NAC) as nutritional supplement to treat psychiatric disorders among which is autism is based on its role as a precursor of glutathione, and its action as a modulating agent of glutamate excitotoxicity, dopaminergic, and inflammatory pathways. Despite the initial promising results in case of NAC, further confirmation in larger samples and with longer follow-up periods is critically needed [12]. Moreover, a larger dose than that used in the randomized clinical trial $(500 \mathrm{mg} /$ day oral over 6 months) might be effective. In spite of my believe that autism is a treatable disorder and that nutritional supplementation as alternative and complementary treatment strategy should not be ignored, and can help to ameliorate the severity of autism (Measured as CARS, SRS, and sensory profile), a critical need for further studies to provide evidence-based guidance to families, clinicians and individuals with autism should be highly encouraged [13].

\section{References}

1. Strati F, Cavalieri D, Albanese D, De Felice C, Donati C, et al. (2017) New evidences on the altered gut microbiota in autism spectrum disorders. Microbiome 5(1): 24.

2. Bjorklund G, Saad K, Chirumbolo S, Kern JK, Geier DA, et al. (2016) Immune dysfunction and neuroinflammation in autism spectrum disorder. Acta Neurobiol Exp (Wars) 76(4): 257-268.
3. El-Ansary A (2016) Data of multiple regressions analysis between selected biomarkers related to glutamate excitotoxicity and oxidative stress in Saudi autistic patients. Data Brief 7: 111-116.

4. El-Ansary A, Hassan WM, Qasem H, Das UN (2016) Identification of Biomarkers of Impaired Sensory Profiles among Autistic Patients. PLoS One 11(11): e0164153.

5. El-Ansary A, Bjørklund G, Chirumbolo S, Alnakhli OM (2017) Predictive value of selected biomarkers related to metabolism and oxidative stress in children with autism spectrum disorder. Metab Brain Dis 32(4): 12091221.

6. Howsmon DP, Kruger U, Melnyk S, James SJ, Hahn J, et al. (2017) Classification and adaptive behavior prediction of children with autism spectrum disorder based upon multivariate data analysis of markers of oxidative stress and DNA methylation. PloS Comput Biol 13(3): e1005385.

7. Diaz Heijtz R, Wang S, Anuar F, Qian Y, Björkholm B, et al. (2011) Normal gut microbiota modulates brain development and behavior. Proc Natl Acad Sci U S A 108(7): 3047-3052.

8. Watson D, O'Connell Motherway M, Schoterman MH, Van Neerven RJ, Nauta A, et al. (2013) Selective carbohydrate utilization by lactobacilli and bifidobacteria. J Appl Microbiol. 114(4): 1132-1146.

9. Hibbeln JR, Linnoila M, Umhau JC, Rawlings R, George DT, et al. (1998) Essential fatty acids predict metabolites of serotonin and dopamine in cerebrospinal fluid among healthy control subjects, and early-and lateonset alcoholics. Biol Psychiatry 44(4): 235-242.

10. Dean OM, Gray KM, Villagonzalo KA, Dodd S, Mohebbi M (2017) A randomised, double blind, placebo-controlled trial of a fixed dose of $\mathrm{N}$-acetyl cysteine in children with autistic disorder. Aust N Z J Psychiatry 51(3): 241-249.

11. Delhey LM, Nur Kilinc E, Yin L, Slattery JC, Tippett ML (2017) The effect of mitochondrial supplements on mitochondrial activity in children with autismspectrum disorder. J Clin Med 6(2).

12. Minarini A, Ferrari S, Galletti M, Giambalvo N, Perrone D, et al. (2017) $\mathrm{N}$-acetylcysteine in the treatment of psychiatric disorders: current status and future prospects. Expert Opin Drug MetabToxicol 13(3): 279-292.

13. El-Ansary A, Al Dera H (2016) Biomarkers directed strategies to treat autism. In: Role of Biomarkers in Medicine. In Tech Publisher, pp. 205228.

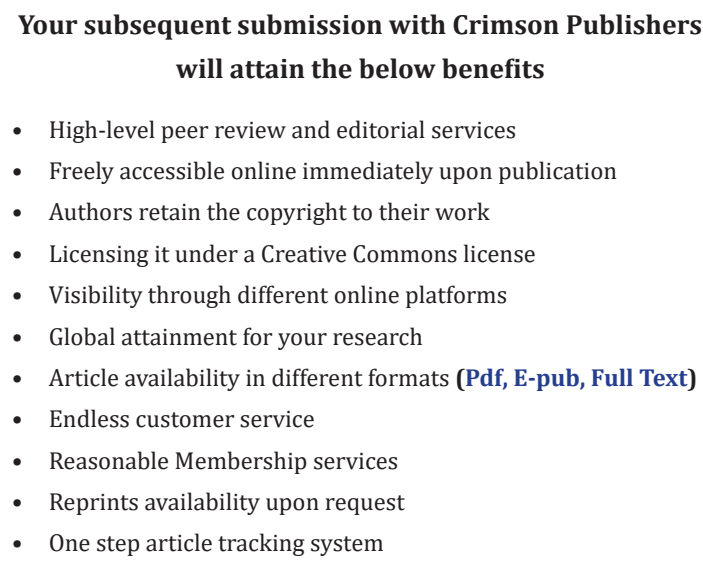

- High-level peer review and editorial services

- Freely accessible online immediately upon publication

- Authors retain the copyright to their work

- Licensing it under a Creative Commons license

- Visibility through different online platforms

- Global attainment for your research

- Article availability in different formats (Pdf, E-pub, Full Text)

- Endless customer service

- Reasonable Membership services

- Reprints availability upon request

- One step article tracking system 